\title{
A Multistage Multiobjective Model for Emergency Evacuation Considering ATIS
}

\author{
Ming-Hua Zeng, ${ }^{1}$ Ke-Jun Long, ${ }^{2}$ Zi-Wen Ling, ${ }^{3}$ and Xi-Yan Huang' \\ ${ }^{1}$ School of Railway Tracks and Transportation, East China Jiaotong University, Nanchang 330013, China \\ ${ }^{2}$ School of Transportation and Traffic Engineering, Changsha University of Science and Technology, Changsha 410075, China \\ ${ }^{3}$ Department of Civil and Environmental Engineering, University of Tennessee-Knoxville, Knoxville, TN 37996-2313, USA
}

Correspondence should be addressed to Xi-Yan Huang; liyan.wong@163.com

Received 26 March 2016; Accepted 12 May 2016

Academic Editor: Yongjun Shen

Copyright (C) 2016 Ming-Hua Zeng et al. This is an open access article distributed under the Creative Commons Attribution License, which permits unrestricted use, distribution, and reproduction in any medium, provided the original work is properly cited.

\begin{abstract}
The impacts of advanced traveler information system's (ATIS's) penetration and compliance rates on network performances during hybrid traffic emergency evacuation are investigated in a degraded road network. Before traffic incident a Path-Size Logit (PSL) route choice model is integrated with constraints on the level of service (LOS) of traffic to formulate a bilevel programming model. It aims at minimizing traffic demand in road network which may locally deteriorate the LOS. The lower level is a PSL-stochastic user equilibrium model for multiple classes of users. During the ongoing incident, a multiobjective multiuser-class stochastic optimization model is established with the objectives of maximizing evacuation reliability and minimizing expected network travel time. Furthermore, computations and analyses are completed for five designated scenarios including a method proposed in previous literature. The results show that the evacuation reliability and different kinds of total expected travel time costs regularly increase with emergency traffic's ATIS compliance rate and decrease with general traffic's ATIS penetration rate. The research will help improve transport network performance when considering ATIS's effect on hybrid traffic.
\end{abstract}

\section{Introduction}

Large city activities and emergent events often add great traffic volumes to an urban road network. The new demand may lead to short-term traffic fluctuation and congestion and should be evacuated in time. This kind of traffic evacuation problem is one of the most important traffic issues around the world.

Researchers have extensively studied evacuation route choice. The main methods can be broadly categorized as static equilibrium assignment [1], dynamic traffic assignment [2], and systematic simulation [3]. Xie and Turnquist [1] studied lane-based evacuation path planning. Brown et al. [2] investigated a strategic hurricane evacuation model based on dynamic traffic assignment. Sansò and Milot [3] proposed a model of three time periods by dividing the time period that contains the time interval during which the traffic accident happened and was sustained into three parts and correspondingly dividing the network state into the equilibrium state before the accident, nonequilibrium state during the accident, and equilibrium state after the accident. Robinson and Khattak [4] integrated a mesoscopic dynamic evacuation transportation simulation and an evacuee route choice decision-making model (representative of the decisions made by potential hurricane evacuees when provided with information on downstream traffic congestion and alternate routes).

In emergent situations caused by, for example, a traffic accident, sudden disorderly increased traffic may lead to degradation or even failure of road segments, which was seldom considered in previous studies. Sumalee and Watling [5] proposed a concept of partial user equilibrium and a corresponding model considering the fact that some travelers recognize the road network degradation. Link degradation possibly brings about local traffic fluctuation and congestion. In order to complete evacuating emergency traffic in a limited time, travel time reliability, an important measurement, should be considered. Capacity degradation will affect the travel time reliability of a road network. Lo and Tung [6] studied the design for transport network with degradable 
links based on reliability analysis. Research on road network reliability focuses on travel time reliability, connectivity reliability, and capacity reliability [7]. In the analysis of reliability, link capacity is often assumed to obey a normal distribution [8] or a uniform distribution $[6,9]$ or other probability distributions. Travel time based reliability measures include travel time reliability [6] and travel time budget reliability [911].

To meet the expectation of coping with traffic problems like emergent degraded road network, advanced traveler information system (ATIS) has been applied widely to public transit and private passenger cars to help improve traffic safety and efficiency. In Robinson and Khattak [4], the integration of a stated-preference-based simulation provided a unique method to forecast ATIS effectiveness, which was assessed by comparing queue sizes and durations on road segments with injected lane closures both with and without the provision of alternative route information. Abundant information may cause network performance deterioration [12] despite benefits from ATIS, so there emerged in previous literature a method of applying equilibrium computation to obtain market penetration rate and compliance rate of these information systems. Penetration rate is expressed as a function of information benefits [13] and compliance rate is calculated by a probability of disutility comparison [14]. We should point out that this does not necessarily lead to optimal network performance. In many circumstances, decision makers wish to obtain optimum transport plans so as to guarantee social efficiency and equity. It is necessary to find out a more effective and efficient way to determine how many ATIS devices are suitable or optimal by exploring other methods. To this important end, we will explore some more different methods to find out a reasonable way to determine the optimal market share of ATIS devices. Since in reality a part of road segments may stochastically degrade due to emergency traffic, this paper will investigate the impact of ATIS (and its penetration rate and compliance rate) and link degradation on the evacuation optimization considering hybrid traffic which consists of evacuation traffic and general traffic.

The remaining parts of this paper are arranged as follows. After describing basic symbols in Section 2, Section 3 presents a Path-Size Logit route choice model, the formulae to calculate penetration rate, compliance rate, and multiuser origindestination (OD) demand. Then the travel time reliability of capacity degraded link was calculated in Section 4. Section 5 establishes multistage, multiuser, and multiobjective evacuation optimization models based on Path-Size Logit(PSL-) stochastic user equilibrium (SUE). Section 6 solves these models and analyzes the impact of penetration and compliance rates of general and evacuation traffic on road network performance and evacuation travel time reliability and the reasonable value of the rates.

\section{Basic Symbols}

Let $\mathbf{G}=(\mathbf{V}, \mathbf{E})$ denote a transport network with a node set $\mathbf{V}$ and a link set $\mathbf{E}$. Let $\mathbf{W}=\{w\}$ be the set of OD pairs, and let $\mathbf{R}_{w}$ be the path set of $w \in \mathbf{W}$. Let $\mathbf{v}=\left(v_{a}\right) \in \mathbb{R}_{+}^{|\mathbf{E}| \times 1}$ be the link flow vector, where $v_{a}$ denotes the traffic flow on road link $a \in \mathbf{E}$ and $\mathbb{R}_{+}$is a set of nonnegative real numbers. Let $\mathbf{f}=\left(f_{r}^{w}\right) \in \mathbb{R}_{+}^{\left(|\mathbf{W}| \cdot\left|\mathbf{R}_{w}\right|\right) \times 1}$ be the path flow vector, where $f_{r}^{w}$ represents the traffic flow on path $r \in \mathbf{R}_{w}$ between OD pair $w$. Let $q_{w}$ and $q_{w^{\prime}}$ (or $q_{w}^{\prime}$ ) denote the OD demand of general traffic between $w \in \mathbf{W}$ and evacuation traffic between $w^{\prime} \in \mathbf{W}$, respectively, where $w^{\prime} \in \mathbf{W}$ is the evacuation OD pair and its corresponding path set is denoted by $\mathbf{R}_{w^{\prime}}$. The OD demand of evacuation traffic can be the demand emerging from certain new OD pairs or can be the new demand flowing between the original OD pairs.

It is assumed that link travel time function $t_{a}\left(v_{a}\right)$ and user disutility (generalized cost) function $u_{a}\left(v_{a}\right)$ are nonnegative, convex, monotonically increasing, and differentiable with $v_{a}$. $t_{a}\left(v_{a}\right)$ is substituted by $u_{a}\left(v_{a}\right)$, since other costs such as fuel cost are not considered in this study. We denote the path disutility between OD pair $w \in \mathbf{W}$ as $u_{r}^{w}$ and calculate it by $u_{r}^{w}=\sum_{a \in \mathbf{E}} u_{a}\left(v_{a}\right) \delta_{r a}^{w}$, where $\delta_{r a}^{w}$ is a dummy variable, $\delta_{r a}^{w}=1$ if link $a$ lies on path $r$, and $\delta_{r a}^{w}=0$ otherwise.

Travelers voluntarily buy and install ATISs. Some of them obey guidance information and others do not. Thus, we assume there are three kinds of users: users of the first class have ATISs and obey guidance information, users of the second class have ATISs but do not obey guidance information, and users of the third class do not have ATISs. The relevant variables of the three user classes such as link travel time, link flow, OD demand, and individual average travel cost $\left(\bar{u}^{w}\right)$ are marked with Arabic numerals 1, 2, and 3 in the lower right corner of corresponding symbols.

\section{Information Utility, Route Choice, and Multiuser OD Demand}

The stochastic degradation of link capacity leads to road users' cognitive bias, so users (maybe particularly those of evacuation traffic) often attempt to reduce such a deviation and then route choice stochasticity by configuring ATISs. It is assumed that the perceptual random errors of path disutility of the three user classes are independent and identically distributed and obey a Gumbel distribution. We adopt the PSL [15] in (1) as the route choice model for the three kinds of users with different stochasticity denoted by positive dispersion parameters $\theta_{i}, i=1,2,3$ :

$$
P_{i r}^{w}=\frac{e^{\mu\left(-\theta_{i} u_{r}^{w}+\ln P S_{r}^{w}\right)}}{\sum_{l \in \mathbf{R}_{w}} e^{\mu\left(-\theta_{i} u_{l}^{w}+\ln P S_{l}^{w}\right)}}, \quad i=1,2,3,
$$

where $\mu$ is a positive parameter (let $\mu=1$ according to usual practice); $\mathrm{PS}_{r}^{w}$ is a path-size attribute, which can be calculated by $\mathrm{PS}_{r}^{w}=\sum_{a \in \mathbf{E}_{r}} L_{a} /\left(L_{r} \sum_{j \in \mathbf{R}_{w}} \delta_{a j}\right)[16]$, where $L_{a}$ and $L_{r}$ are the lengths of link $a$ and path $r$; $\mathbf{E}_{r}$ is the set of links on path $r$ between OD pair $w$. The length of link or path is measured by its disutility. The relatively simple PSL model was proposed by Ben-Akiva and Bierlaire [15]; it can overcome the theoretical deficits of C-Logit model and can capture route overlapping. 
Given route probability $P_{i r}^{w}$, the individual average travel cost $\bar{u}_{i}^{w}(i=1,2,3)$ of each user class is calculated by

$$
\bar{u}_{i}^{w}=\sum_{r \in \mathbf{R}_{w}} u_{r}^{w} P_{i r}^{w}, \quad \forall i=1,2,3, w \in \mathbf{W} .
$$

Thus, the individual average disutilities $\bar{u}^{w \text {,equ }}$ and $\bar{u}^{w \text {, unequ }}$ of user class using ATIS and without ATIS are, respectively,

$$
\begin{aligned}
\bar{u}^{w \text {, equ }} & =\varphi_{w} \bar{u}_{1}^{w}+\left(1-\varphi_{w}\right) \bar{u}_{2}^{w}, \\
\bar{u}^{w \text {,unequ }} & =\bar{u}_{3}^{w},
\end{aligned}
$$

where $\varphi_{w}$ is the compliance rate of the first class user (correspondingly, denote the ATIS compliance rate of emergency traveler in the first class as $\varphi_{w}^{\prime}$ ) and it can be calculated as follows:

$$
\begin{aligned}
& \varphi_{w}= \operatorname{Pr}\left(U_{1}^{w} \leq U_{2}^{w}\right) \\
&=\sum_{\text {all } u_{2}^{w}} \operatorname{Pr}\left(U_{1}^{w} \leq u_{2}^{w}\right) \operatorname{Pr}\left(U_{2}^{w}=u_{2}^{w}\right) \sum_{r \in \mathbf{R}_{w}} \sum_{k \in \mathbf{R}_{w}^{r}} P_{2 r}^{w} P_{1 k}^{w}, \\
& \forall w \in \mathbf{W} .
\end{aligned}
$$

In (4), $U_{1}^{w}$ and $U_{2}^{w}$ are independent random variables, and $\mathbf{R}_{w}^{r}$ is the path set: $\mathbf{R}_{w}^{r}=\left\{l \mid u_{l}^{w} \leq u_{r}^{w}\right\}$.

It is assumed that ATISs are allocated to all evacuation traffic. The ATIS penetration rates $\lambda_{w} \in(0,1)$ of OD pair $w$ are partially determined by information benefits, that is, the disutility difference between general traffic with ATIS and users without ATIS; namely,

$$
\lambda_{w}=\frac{1}{1+\exp \left(a_{\lambda}+b_{\lambda}\left(\bar{u}^{w \text {,equ }}-\bar{u}^{w, \text {,unequ }}\right)\right)},
$$

where $a_{\lambda}>0$ and $b_{\lambda}>0$ are constant and can be set as $a_{\lambda}=$ 1.75 and $b_{\lambda}=0.5$ [14].

Hence, in terms of the formulae of penetration and compliance rates, we obtain OD demand for each user category as follows:

$$
\begin{aligned}
& q_{1 w}=\varphi_{w}^{\prime} q_{w}^{\prime}+q_{w} \lambda_{w} \varphi_{w} \\
& q_{2 w}=\left(1-\varphi_{w}^{\prime}\right) q_{w}^{\prime}+q_{w} \lambda_{w}\left(1-\varphi_{w}\right) \\
& q_{3 w}=q_{w}\left(1-\lambda_{w}\right)
\end{aligned}
$$

\section{Travel Time Reliability of Capacity Degraded Link}

It is assumed that some link capacities are degradable due to stochastic factors such as traffic incident and that the actual capacity of a road link $C_{a}$ is a random variable. Furthermore, we suppose that $C_{a}$ obeys a uniform distribution [6] in the interval $\left[\vartheta_{a} \bar{c}_{a}, \bar{c}_{a}\right]$, where $\bar{c}_{a}$ is link design capacity and $\vartheta_{a} \in$ $[0,1)$ is worst capacity utilization coefficient reflecting the utilization rate of link capacity as transport system conditions worsen.
The well-known US Bureau of Public Roads (BPR) function is adopted to calculate link travel time:

$$
t_{a}\left(x_{a}, C_{a}\right)=t_{a}^{0}\left(1+0.15\left(\frac{x_{a}}{C_{a}}\right)^{4}\right),
$$

where $t_{a}^{0}$ is free flow travel time on ink $a$. Assuming the mutual independence of link flow $x_{a}$ and capacity $C_{a}$, we can calculate expected link time as follows:

$$
E\left(t_{a}\left(x_{a}, C_{a}\right)\right)=t_{a}^{0}+0.15 t_{a}^{0} x_{a}^{4} \frac{1-\vartheta_{a}^{-3}}{-3 \bar{c}_{a}^{4}\left(1-\vartheta_{a}\right)}
$$

Bell and Iida [17] referred to travel time reliability as the probability to complete a travel during the expected period of time under a specific OD demand. We define travel time reliability as $R_{a}\left(x_{a}\right)=\operatorname{Pr}\left\{t_{a}\left(x_{a}\right) \leq \Phi_{a} t_{a}^{0}\right\}$, where $\Phi_{a}>1$ is an acceptable congestion level for travelers on degraded road link $a$. Hence, let $\Phi_{a}^{\prime}=\left(\left(\Phi_{a}-1\right) / 0.15\right)^{1 / 4}$; we can derive a direct expression for $R_{a}\left(x_{a}\right)$ as follows:

$$
\begin{gathered}
R_{a}\left(x_{a}\right)=\operatorname{Pr}\left\{C_{a} \geq \frac{x_{a}}{\Phi_{a}^{\prime}}\right\}=1-\operatorname{Pr}\left\{C_{a}<\frac{x_{a}}{\Phi_{a}^{\prime}}\right\} \\
\quad=\left\{\begin{array}{lc}
1, & x_{a} \leq \Phi_{a}^{\prime} \vartheta_{a} \bar{c}_{a} ; \\
1-\frac{x_{a}-\Phi_{a}^{\prime} \vartheta_{a} \bar{c}_{a}}{\Phi_{a}^{\prime} \bar{c}_{a}\left(1-\vartheta_{a}\right)}, & \Phi_{a}^{\prime} \vartheta_{a} \bar{c}_{a}<x_{a} \leq \Phi_{a}^{\prime} \bar{c}_{a} ; \\
0, & x_{a}>\Phi_{a}^{\prime} \bar{c}_{a} .
\end{array}\right.
\end{gathered}
$$

\section{Multilevel Multiobjective Mathematical Evacuation Models Based on Hybrid Traffic SUE}

We assume that the traffic incident happens on the road link $a_{I} \in \mathbf{E}$. The capacity of this road link is assumed to be declined to $\sigma \bar{c}_{a}$, where $\sigma \in(0,1)$ is a small positive number. Based on the PSL-SUE model and the constraint of level of service (LOS), a bilevel programming (BP) model shown as (10) and (11) is established to minimize traffic demand under local deterioration of LOS. Consider

$$
\begin{array}{ll}
\min & \varepsilon \\
\text { s.t. } & \frac{v_{a}(\varepsilon \mathbf{q})}{\bar{c}_{a}} \geq \operatorname{LOS}_{0}, \quad \exists a \in \mathbf{E},
\end{array}
$$

where $\varepsilon>0$ is the scale factor of traffic demand; $\operatorname{LOS}_{0}>$ 0 is the threshold of LOS. Link flow $v_{a}(\varepsilon \mathbf{q})$ can be obtained 
by solving the following lower level multiuser traffic network equilibrium model:

$$
\begin{array}{ll}
\min \quad & Z_{1}(f) \\
& =\sum_{a \in \mathbf{E}} \int_{0}^{v_{a}} t_{a}\left(y, \bar{c}_{a}\right) d y \\
& +\sum_{i=1}^{3} \frac{1}{\theta_{i}}\left(\sum_{w \in \mathbf{W}} \sum_{r \in \mathbf{R}_{w}} f_{i r}^{w} \ln f_{i r}^{w}-\sum_{w \in \mathbf{W}} \sum_{r \in \mathbf{R}_{w}} f_{i r}^{w} \mathrm{PS}_{i r}^{w}\right) \\
\text { s.t. } \quad \sum_{r \in \mathbf{R}_{w}} f_{i r}^{w}=q_{i w}, \quad \forall w \in \mathbf{W} & \\
& q_{1 w}+q_{2 w}+q_{3 w}=(1+\varepsilon) q_{w}, \quad \forall r \in \mathbf{R}_{w}, w \in \mathbf{W} \\
& v_{a}=\sum_{w \in \mathbf{W}} \sum_{r \in \mathbf{R}_{w}}\left(\sum_{i=1}^{3} f_{i r}^{w}\right) \delta_{a r}^{w}, \quad \forall a \in \mathbf{E} \\
& f_{i r}^{w} \geq 0, \quad \forall i=1,2,3, r \in \mathbf{R}_{w}, w \in \mathbf{W},
\end{array}
$$

$$
\begin{aligned}
\max \quad Z_{R, E E} & =\sum_{w^{\prime} \in \mathbf{W}} R_{w, E E}^{\prime} \\
\min \quad Z_{2}(f) & \\
= & \sum_{a \in \mathbf{E}} \int_{0}^{v_{a}} E\left(t_{a}\left(y, C_{a}\right)\right) d y+\sum_{i=1}^{3} \frac{1}{\theta_{i}}\left(\sum_{w\left(\neq w^{\prime}\right) \in \mathbf{W}} \sum_{r \in \mathbf{R}_{w}} f_{i r}^{w} \ln f_{i r}^{w}-\sum_{w\left(\neq w^{\prime}\right) \in \mathbf{W}} \sum_{r \in \mathbf{R}_{w}} f_{i r}^{w} \mathrm{PS}_{i r}^{w}\right) \\
& +\sum_{i=1}^{3} \frac{1}{\theta_{i}^{\prime}}\left(\sum_{w^{\prime} \in \mathbf{W}} \sum_{r \in \mathbf{R}_{w^{\prime}}} f_{i r}^{w^{\prime}} \ln f_{i r}^{w^{\prime}}-\sum_{w^{\prime} \in \mathbf{W}} \sum_{r \in \mathbf{R}_{w^{\prime}}} f_{i r}^{w^{\prime}} \mathrm{PS}_{i r}^{w^{\prime}}\right)
\end{aligned}
$$$$
\text { s.t. } \quad \sum_{r \in \mathbf{R}_{w} \cup \mathbf{R}_{w^{\prime}}} f_{i r}^{w}=q_{i w}, \quad \forall w, w^{\prime} \in \mathbf{W}, i=1,2,3
$$$$
v_{a}=\sum_{w \in \mathbf{W}} \sum_{r \in \mathbf{R}_{w}}\left(\sum_{i=1}^{3} f_{i r}^{w}\right) \delta_{a r}^{w}+\sum_{w^{\prime} \in \mathbf{W}} \sum_{r \in \mathbf{R}_{w^{\prime}}}\left(\sum_{i=1}^{3} f_{i r}^{w^{\prime}}\right) \delta_{a r}^{w^{\prime}}, \quad \forall a \in \mathbf{E}
$$$$
f_{i r}^{w} \geq 0, \quad \forall r \in \mathbf{R}_{w}, w \in \mathbf{W}, \quad i=1,2,3
$$$$
f_{i r}^{w^{\prime}} \geq 0, \quad \forall r \in \mathbf{R}_{w^{\prime}}, w^{\prime} \in \mathbf{W}, \quad i=1,2,3,
$$

where dispersion parameters $\theta_{1}, \theta_{2}$, and $\theta_{3}\left(\theta_{1}>\theta_{2}>\theta_{3}\right)$ represent the route choice stochasticity of the three traveler categories.

Before the traffic incident, the BP model is solved to get the link flow distribution of OD demand $\mathbf{q}$ and further to determine capacity degradation for the network. After the traffic incident, link capacity declines stochastically; the degradable links are obtained by the aforementioned bilevel programming. Through the duration of the traffic incident, a part of travelers who have obtained traffic information change their travel routes due to the reunderstanding of link capacity drop and the change of network state. During the sustaining time of incident, network traffic assignment and optimization are completed based on the solution to BP model through the following multiobjective mathematical programming (MOMP). The MOMP is proposed as formulae in (12). The MOMP aims to maximize travel time reliability of evacuation traffic through Path-Size Logit-stochastic equilibrium assignment for $q_{w}$ and $q_{w}^{\prime}$ in the degradable network. Consider where $\theta_{i}^{\prime}$ and $\theta_{i}, i=1,2,3$, are dispersion parameters and satisfy $\theta_{i}^{\prime} \leq \theta_{i}, i=1,2,3$, and $\theta_{1}^{\prime}>\theta_{2}^{\prime}>\theta_{3}^{\prime}$. The first objective function, travel time reliability of evacuation traffic $Z_{R, E E}$, is the sum of evacuation OD travel time reliability $R_{w, E E}^{\prime}=$ $1-\prod_{r \in \mathbf{R}_{w^{\prime}}}\left(1-R_{r}^{w^{\prime}}\right), w^{\prime} \in \mathbf{W}$, where $R_{r}^{w^{\prime}}=\prod_{a \in \mathbf{E}_{r}^{w^{\prime}}} R_{a}, \forall r \in$ $\mathbf{R}_{w^{\prime}}, w^{\prime} \in \mathbf{W}$, is path travel time reliability.

\section{Numerical Computation and Analyses}

The example network used for case study is shown as in Figure 1. The digit in the circle represents node number. The digit beside the link (arrow line) represents link number.
Link free flow travel time $t_{a}^{0}$ and capacity $\bar{c}_{a}$ are shown as in Table 1. There are two pairs of OD, that is, one general traffic OD pair $(1,9)$ and one evacuation traffic OD pair $(2,9)$. The emergency evacuation traffic volume is $q_{(2,9)}=300$. It is assumed that a traffic incident happens in link 4. Between any OD pair, path sequences represented by node number are listed in Table 2.

The values of parameters and initial values of variables are set as follows. Set parameter values $\Phi_{a}=1.2, \sigma=10 \%$, $\sigma_{q}=26 \%, \operatorname{LOS}_{0}=1.2$, and $\varepsilon_{1}=\varepsilon_{2}=\varepsilon_{3}=0.001$, and let $\theta_{1}^{\prime}=\theta_{1}=1, \theta_{2}^{\prime}=\theta_{2}=0.1$, and $\theta_{3}^{\prime}=\theta_{3}=0.01$ without differentiating the dispersion parameters of $\mathrm{BP}$ and MOMP. Set initial values $q_{(1,9)}^{(0)}=300, \varphi_{w}^{(0)}=0.3$, and 
TABLE 1: FFTT and capacities of links.

\begin{tabular}{lcccccccccccc}
\hline Link number & 1 & 2 & 3 & 4 & 5 & 6 & 7 & 8 & 9 & 10 & 11 \\
\hline$t_{a}^{0}(\mathrm{~h})$ & 0.125 & 0.125 & 0.125 & 0.1 & 0.125 & 0.1 & 0.1 & 0.125 & 0.1 & 0.125 & 0.125 & 0.125 \\
$\bar{c}_{a}(\mathrm{veh})$ & 350 & 280 & 350 & 500 & 300 & 500 & 500 & 280 & 500 & 220 & 300 & 220 \\
\hline
\end{tabular}

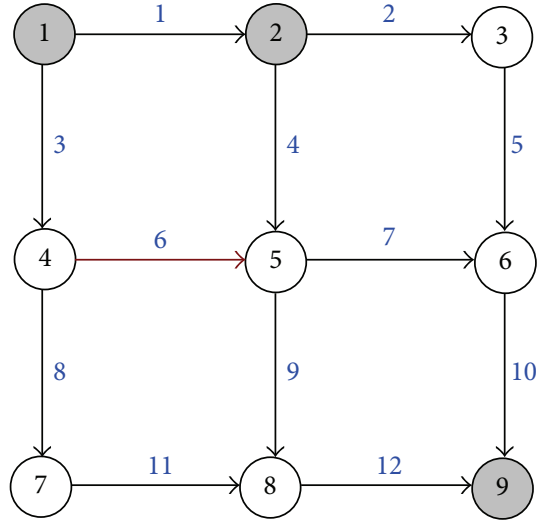

FIgURE 1: Example network.

TABle 2: Path sequences of general and emergency traffic.

\begin{tabular}{lc}
\hline Path number & Path sequences \\
\hline 1 & $1-2-3-6-9$ \\
2 & $1-4-7-8-9$ \\
3 & $1-2-5-6-9$ \\
4 & $1-4-5-8-9$ \\
5 & $1-2-5-8-9$ \\
6 & $1-4-5-6-9$ \\
7 & $2-3-6-9$ \\
8 & $2-5-6-9$ \\
9 & $2-5-8-9$ \\
\hline
\end{tabular}

$\lambda_{w}^{(0)}=0.9$, and let evacuation compliance rate $\varphi_{w}^{\prime(0)}=1$ if this rate is determined endogenously. The multiple objectives are summed using linear weighted method. The weight vector, composed of weight of expected path travel time and that of the inverse of evacuation reliability, is given as $(0.87,0.13)$. It should be pointed out that all the parameter values and initial variable values are fixed except special requirement in the following numerical studies.

We design five scenarios, which define different approaches to getting ATIS penetration rate and compliance rate shown as in Table 3, to solve models. All evacuation traffic is supposed to be deployed with ATIS systems in scenarios $A \sim D$.

For SA SE, at the first stage, the model expressed by (10) and (11) is solved after three times of iteration and generate $\varepsilon_{\text {min }}=1$. Thus, we obtain the general traffic demand $q_{(1,9)}=$ 600 and only the fourth link $(2,5)$ is degradable.

Link capacity degradation caused by demand growth or fluctuation in traffic incident can affect road network performance. When worst capacity utilization coefficients $\vartheta_{a}(a \in \mathbf{E})$ get different values, ATIS penetration and
TABLE 3: Five scenarios about ATIS rates.

\begin{tabular}{lcc}
\hline & Rates fixed & $\begin{array}{c}\text { Rates obtained by } \\
\text { equilibrium } \\
\text { computation }\end{array}$ \\
\hline Scenario A (SA) & $\varphi_{w}^{\prime}=1$ & $\lambda_{w}$ and $\varphi_{w}$ \\
Scenario B (SB) & $\varphi_{w}^{\prime}=1, \lambda_{w}=$ & $\varphi_{w}$ \\
& $0.1,0.2, \ldots, 0.9,1.0$ & ${ }^{\prime}=$ \\
Scenario C (SC) & $0.1,0.2, \ldots, 0.9,1.0$ & $\lambda_{w}$ and $\varphi_{w}$ \\
Scenario D (SD) & $0.1,0.2, \ldots, 0.9,1.0$, & $\varphi_{w}^{\prime}=$ \\
& $\lambda_{w}=0.1$ & $\varphi_{w}$ \\
Scenario E (SE) & Not fixed & $\lambda_{w}^{\prime}, \varphi_{w}^{\prime}, \lambda_{w}$, and $\varphi_{w}$ \\
\hline
\end{tabular}

compliance rates (both are calculated in SA, SC, and SE) will come to different results which will lead to different network flow distributions and performance index values. Given $\vartheta_{(2,5)}=0.7$, we solve model (12) and get results as shown in Tables $4-8$. Different values of $\vartheta_{(2,5)}$ are tested and their corresponding results do not affect our conclusions.

As to the network flow distribution, the flow pattern of all paths $\left(f_{r}^{w}+f_{r}^{w^{\prime}}\right)$ and the link flow pattern of evacuation traffic $\left(v_{a, E E}\right)$ are shown in Table 4 . Computational results show that $\left(f_{r}^{w}+f_{r}^{w^{\prime}}\right)$ and $\left(v_{a, E E}\right)$ obtained from all cases in scenarios $\mathrm{A} \sim \mathrm{E}$ are similar except for slight fluctuations.

Penetration and compliance rates obtained by equilibrium computation in SA are $\lambda_{w}=0.1481$ and $\varphi_{w}=0.5956$, and the corresponding evacuation reliability is $R_{w, E E}^{\prime}=$ 0.2036 , while penetration and compliance rates obtained by equilibrium computation in $\mathrm{SB}$ are $\lambda_{w}^{\prime}=0.1482, \varphi_{w}^{\prime}=0.6794$, $\lambda_{w}=0.1482$, and $\varphi_{w}=0.5980$, and the corresponding evacuation reliability is $R_{w, E E}^{\prime}=0.2258$. Obviously, when the rates of penetration and compliance for evacuation traffic and general traffic are endogenously determined, the reliability of evacuation traffic is improved compared to the situation where only the rates for general traffic are endogenously determined.

In scenario $\mathrm{B}$, the $\mathrm{OD}$ reliability of evacuation traffic $R_{w, E E}^{\prime}$ improves with the penetration rate of general traffic $\lambda_{w}$; that is, $R_{w, E E}^{\prime} \propto \lambda_{w}$. The TETTCs of the whole network, evacuation traffic, and the third user class decrease with $\lambda_{w}$; that is, $R_{w, E E}^{\prime} \propto 1 / \lambda_{w}$. From microscopic perspective, the LOS of the most congested road link number 10 is slightly enhanced, though those of the mild congested road links numbers 4, 9, and 12 are slightly declined.

In scenario $\mathrm{C}$, if the compliance rate of evacuation traffic $\varphi_{w}^{\prime}$ gets larger value, then the penetration rate of general traffic $\lambda_{w}$ maintains the same, compliance rate of general traffic $\varphi_{w}$ gets very slight decline, and the OD reliability of 
TABLE 4: Computational results for SA.

\begin{tabular}{|c|c|c|c|c|c|c|c|c|c|}
\hline & $\lambda_{w}$ & $\varphi_{w}$ & $R_{w, E E}^{\prime}$ & $\begin{array}{l}\text { Whole } \\
\text { network }\end{array}$ & $\begin{array}{l}\text { Evacuation } \\
\text { traffic }\end{array}$ & $\begin{array}{c}\text { TETTC } \\
\text { Users of } \\
\text { class } 1\end{array}$ & $\begin{array}{l}\text { Users of } \\
\text { class } 2\end{array}$ & $\begin{array}{c}\text { Users of } \\
\text { class } 3\end{array}$ & \\
\hline & 0.1481 & 0.5956 & 0.2036 & 482.3278 & 139.7833 & 231.9199 & 10.2859 & 240.1219 & \\
\hline Path number & 1 & 2 & 3 & 4 & 5 & 6 & 7 & 8 & 9 \\
\hline$f_{r}^{w}+f_{r}^{w^{\prime}}$ & 98.0117 & 101.2941 & 98.4531 & 102.0609 & 100.8507 & 99.3295 & 95.7343 & 97.3151 & 106.9506 \\
\hline Link number & 2 & 4 & 5 & 7 & 9 & 10 & 12 & & \\
\hline$v_{a, E E}$ & 95.7343 & 204.2657 & 95.7343 & 97.3151 & 106.9506 & 193.0494 & 106.9506 & & \\
\hline
\end{tabular}

Please note that $v_{a, E E}$ represents the evacuation traffic flow on link $a$.

TABLE 5: Computational results for SB.

\begin{tabular}{|c|c|c|c|c|c|c|c|c|c|c|}
\hline \multirow{2}{*}{$\lambda_{w}$} & \multirow{2}{*}{$R_{w, E E}^{\prime}$} & \multicolumn{4}{|c|}{ TETTC } & \multicolumn{5}{|c|}{$\begin{array}{l}\text { Link V/C ratios larger than one for } \\
\text { optimum solution }\end{array}$} \\
\hline & & $\begin{array}{l}\text { Whole } \\
\text { network }\end{array}$ & $\begin{array}{c}\text { Evacuation } \\
\text { traffic }\end{array}$ & $\begin{array}{c}\text { Users of } \\
\text { class } 1\end{array}$ & $\begin{array}{c}\text { Users of } \\
\text { class } 2\end{array}$ & $\begin{array}{c}\text { Users of } \\
\text { class } 3\end{array}$ & Link 4 & Link 9 & Link 10 & Link 12 \\
\hline 0.00 & 0.1927 & 484.0581 & 140.2266 & 140.2266 & 0.0000 & 343.8315 & 1.0125 & 1.0281 & 1.6384 & 1.0212 \\
\hline 0.10 & 0.1953 & 483.2172 & 139.9648 & 170.6846 & 3.4324 & 309.1002 & 1.0116 & 1.0311 & 1.6337 & 1.0247 \\
\hline 0.20 & 0.1979 & 482.4337 & 139.7201 & 201.1091 & 6.8578 & 274.4667 & 1.0108 & 1.0340 & 1.6293 & 1.0280 \\
\hline 0.30 & 0.2003 & 481.7007 & 139.4899 & 231.5024 & 10.2768 & 239.9215 & 1.0100 & 1.0368 & 1.6251 & 1.0312 \\
\hline 0.40 & 0.2026 & 481.0099 & 139.2710 & 261.8647 & 13.6899 & 205.4553 & 1.0093 & 1.0394 & 1.6211 & 1.0342 \\
\hline 0.50 & 0.2048 & 480.3628 & 139.0649 & 292.2022 & 17.0977 & 171.0630 & 1.0086 & 1.0420 & 1.6172 & 1.0371 \\
\hline 0.60 & 0.2068 & 479.7560 & 138.8707 & 322.5175 & 20.5007 & 136.7378 & 1.0079 & 1.0444 & 1.6136 & 1.0398 \\
\hline 0.70 & 0.2088 & 479.1810 & 138.6850 & 352.8085 & 23.8992 & 102.4733 & 1.0073 & 1.0468 & 1.6100 & 1.0425 \\
\hline 0.80 & 0.2106 & 478.6305 & 138.5052 & 383.0728 & 27.2933 & 68.2644 & 1.0067 & 1.0491 & 1.6066 & 1.0451 \\
\hline 0.90 & 0.2124 & 478.1115 & 138.3348 & 413.3198 & 30.6836 & 34.1080 & 1.0061 & 1.0513 & 1.6033 & 1.0475 \\
\hline 1.00 & 0.2140 & 477.6171 & 138.1713 & 443.5467 & 34.0704 & 0.0000 & 1.0056 & 1.0535 & 1.6001 & 1.0499 \\
\hline
\end{tabular}

evacuation traffic $R_{w, E E}^{\prime}$ becomes worse. The TETTCs of the whole network, evacuation traffic, and users of class 3 drop very little which is similar to scenario B; the TETTC of user class 2 gets sharp drop, while the TETTC of user class 1 gets substantial increase. The results of scenario D are similar to scenario C.

\section{Conclusions}

This paper investigates a multistage multiobjective evacuation optimization problem for multiuser hybrid traffic under the stochastic degradation of road network resulting from traffic incident. We construct bilevel programming and multiobjective programming and carry out series of computational analyses with some useful conclusions.

Expecting to clean the traffic incident spot as quickly as possible, each emergency user is assumed to be installed with ATIS. Given ATIS penetration and compliance rates of evacuation traffic and compliance rate of general traffic, the travel time reliability of evacuation traffic increases with ATIS penetration rate of general traffic; given evacuation penetration rate and endogenously computed general penetration and compliance rates, the travel time reliability of evacuation traffic decreases with evacuation compliance rate. If general traffic penetration rate increases, then the total expected travel time cost of the first user class gets a significant rise, that of the second user class rises, that of the third user class gets a sharp drop, and that of the whole network and evacuation traffic drops a little; if evacuation traffic penetration rate increases, then the total expected travel time cost of the first user class increases significantly, while that of the second user class gets a sharp drop, and the other kinds of total expected travel time cost drop slightly.

In literature that does not consider ATIS penetration and compliance rates of evacuation traffic, scenario $\mathrm{E}$ is the most traditional way, for example, [14]. In this paper, we consider emergency evacuation, add evacuation travel time reliability to establish the multiobjective optimization model, and design other four scenarios for equilibrium computation. Intensive investigations show that, in scenario $\mathrm{E}$, the evacuation travel time reliability is not the maximum one and the total expected travel time cost of the whole network is not the smallest among the five scenarios. The designed scenarios can obtain the maximum evacuation travel time reliability and the minimum total expected travel time cost of the whole hybrid transport network, which are attained by computation results of link flow pattern and ATIS rates that will help reasonably plan emergency traffic travel routes, guide nonemergency social traffic, and adjust ATIS utilization. Findings in this study also can assist policy makers and emergency management department to make more comprehensive planning and make decisions. Further study 
TABLE 6: Computational results for SC.

\begin{tabular}{|c|c|c|c|c|c|c|c|c|}
\hline \multirow{2}{*}{$\varphi_{w}^{\prime}$} & \multirow{2}{*}{$\lambda_{w}$} & \multirow{2}{*}{$\varphi_{w}$} & \multirow{2}{*}{$R_{w, E E}^{\prime}$} & \multicolumn{5}{|c|}{ TETTC } \\
\hline & & & & Whole network & Evacuation traffic & Users of class 1 & Users of class 2 & Users of class 3 \\
\hline 0.00 & 0.1482 & 0.5983 & 0.2331 & 484.4268 & 140.1594 & 92.2212 & 151.4444 & 240.7612 \\
\hline 0.10 & 0.1482 & 0.5981 & 0.2294 & 484.0956 & 140.0788 & 106.2035 & 137.2321 & 240.6599 \\
\hline 0.20 & 0.1482 & 0.5980 & 0.2258 & 483.7846 & 140.0046 & 120.1735 & 123.0463 & 240.5648 \\
\hline 0.30 & 0.1482 & 0.5979 & 0.2223 & 483.4867 & 139.9329 & 134.1302 & 108.8830 & 240.4735 \\
\hline 0.40 & 0.1482 & 0.5978 & 0.2189 & 483.2007 & 139.8635 & 148.0741 & 94.7410 & 240.3856 \\
\hline 0.50 & 0.1482 & 0.5977 & 0.2156 & 482.9319 & 139.7992 & 162.0087 & 80.6203 & 240.3030 \\
\hline 0.60 & 0.1482 & 0.5976 & 0.2124 & 482.6674 & 139.7338 & 175.9289 & 66.5172 & 240.2213 \\
\hline 0.70 & 0.1482 & 0.5975 & 0.2093 & 482.4126 & 139.6702 & 189.8380 & 52.4321 & 240.1426 \\
\hline 0.80 & 0.1482 & 0.5974 & 0.2062 & 482.1669 & 139.6085 & 203.7363 & 38.3642 & 240.0664 \\
\hline 0.90 & 0.1482 & 0.5973 & 0.2032 & 481.9298 & 139.5484 & 217.6243 & 24.3127 & 239.9928 \\
\hline 1.00 & 0.1482 & 0.5972 & 0.2003 & 481.7007 & 139.4899 & 231.5024 & 10.2768 & 239.9215 \\
\hline
\end{tabular}

TABLE 7: Computational results for SD.

\begin{tabular}{|c|c|c|c|c|c|c|c|}
\hline \multirow{2}{*}{$\varphi_{w}^{\prime}$} & \multirow{2}{*}{$\lambda_{w}$} & \multirow{2}{*}{$R_{w, E E}^{\prime}$} & \multicolumn{5}{|c|}{ TETTC } \\
\hline & & & Whole network & Evacuation traffic & Users of class 1 & Users of class 2 & Users of class 3 \\
\hline 0.00 & 0.1 & 0.2295 & 486.3377 & 140.6514 & 30.7854 & 145.2223 & 310.3299 \\
\hline 0.10 & 0.1 & 0.2254 & 485.9627 & 140.5864 & 44.8392 & 130.9395 & 310.1841 \\
\hline 0.20 & 0.1 & 0.2216 & 485.6097 & 140.5200 & 58.8790 & 116.6844 & 310.0463 \\
\hline 0.30 & 0.1 & 0.2179 & 485.2747 & 140.4530 & 72.9050 & 102.4546 & 309.9152 \\
\hline 0.40 & 0.1 & 0.2144 & 484.9501 & 140.3826 & 86.9155 & 88.2471 & 309.7876 \\
\hline 0.50 & 0.1 & 0.2110 & 484.6349 & 140.3104 & 100.9105 & 74.0612 & 309.6632 \\
\hline 0.60 & 0.1 & 0.2077 & 484.3330 & 140.2400 & 114.8922 & 59.8968 & 309.5439 \\
\hline 0.70 & 0.1 & 0.2045 & 484.0386 & 140.1688 & 128.8591 & 45.7523 & 309.4272 \\
\hline 0.80 & 0.1 & 0.2013 & 483.7558 & 140.0996 & 142.8135 & 31.6274 & 309.3148 \\
\hline 0.90 & 0.1 & 0.1983 & 483.4837 & 140.0324 & 156.7561 & 17.5211 & 309.2065 \\
\hline 1.00 & 0.1 & 0.1953 & 483.2172 & 139.9648 & 170.6846 & 3.4324 & 309.1002 \\
\hline
\end{tabular}

TABLE 8: Computational results for SE.

\begin{tabular}{lccccccccc}
\hline$\lambda_{w}^{\prime}$ & $\varphi_{w}^{\prime}$ & $\lambda_{w}$ & $\varphi_{w}$ & $R_{w, E E}^{\prime}$ & Whole network & Users of class 1 & Users of class 2 & Users of class 3 \\
0.1482 & 0.6794 & 0.1482 & 0.5980 & 0.2258 & 483.7801 & 129.9732 & 14.5312 & 339.2757 \\
\hline Path number & 1 & 2 & 3 & 4 & 5 & 6 & 7 & 8 \\
$f_{r}^{w}+f_{r}^{w^{\prime}}$ & 97.5855 & 101.5892 & 98.1286 & 102.5242 & 101.0878 & 99.0847 & 98.5036 & 99.0493 & 102.4471 \\
\hline Link number & 2 & 4 & 5 & 7 & 9 & 10 & 12 & \\
$v_{a, E E}$ & 94.6516 & 205.3484 & 94.6516 & 96.6016 & 108.7468 & 191.2532 & 108.7468 \\
\hline
\end{tabular}

will focus on optimal designation for degradable transport network with hybrid traffic considering the impact of the market share of advanced information and its distribution in network.

\section{Competing Interests}

The authors declare that there are no competing interests regarding the publication of this paper.

\section{Acknowledgments}

This research was supported by Projects of the National Natural Science Foundation of China (Grant no. 51468020),
Natural Science Foundation of Jiangxi Province of China (Grant no. 20142BAB207016), Science and Technology Support Program of Jiangxi Province (Grant no. 20151BBG70056), and Science and Technology Project of Jiangxi Provincial Department of Transportation (no. 2013C0008 and no. 2014X0014).

\section{References}

[1] C. Xie and M. A. Turnquist, "Lane-based evacuation network optimization: an integrated Lagrangian relaxation and tabu search approach," Transportation Research Part C: Emerging Technologies, vol. 19, no. 1, pp. 40-63, 2011. 
[2] C. Brown, W. White, C. Van Slyke, and J. D. Benson, "Development of a strategic hurricane evacuation-dynamic traffic assignment model for the Houston, Texas, region," Transportation Research Record, vol. 2137, pp. 46-53, 2009.

[3] B. Sansò and L. Milot, "Performability of a congested urban transportation network when accident information is available," Transportation Science, vol. 33, no. 1, pp. 68-79, 1999.

[4] R. Robinson and A. Khattak, "Evacuee route choice decisions in a dynamic hurricane evacuation context," Transportation Research Record, vol. 2312, no. 15, pp. 141-149, 2012.

[5] A. Sumalee and P. D. Watling, "Travel time reliability in a network with dependent link modes and partial driver response," Journal of the Eastern Asia Society for Transportation Studies, vol. 5, pp. 1687-1701, 2003.

[6] H. K. Lo and Y.-K. Tung, "Network with degradable links: capacity analysis and design," Transportation Research, Part B: Methodological, vol. 37, no. 4, pp. 345-363, 2003.

[7] D.-H. Wang, H.-S. Qi, and C. Xu, "Reviewing traffic reliability research," Journal of Transportation Systems Engineering and Information Technology, vol. 10, no. 5, pp. 12-21, 2010.

[8] W. H. K. Lam, H. Shao, and A. Sumalee, "Modeling impacts of adverse weather conditions on a road network with uncertainties in demand and supply," Transportation Research Part B: Methodological, vol. 42, no. 10, pp. 890-910, 2008.

[9] H. K. Lo, X. W. Luo, and B. W. Y. Siu, "Degradable transport network: travel time budget of travelers with heterogeneous risk aversion," Transportation Research Part B: Methodological, vol. 40, no. 9, pp. 792-806, 2006.

[10] A. Chen, P. Kasikitwiwat, and C. Yang, "Alternate capacity reliability measures for transportation networks," Journal of Advanced Transportation, vol. 47, no. 1, pp. 79-104, 2013.

[11] W. Y. Szeto, Y. Jiang, K. I. Wong, and M. Solayappan, "Reliability-based stochastic transit assignment with capacity constraints: formulation and solution method," Transportation Research Part C: Emerging Technologies, vol. 35, pp. 286-304, 2013.

[12] M. Ben-Akiva, A. De Palma, and K. Isam, "Dynamic network models and driver information systems," Transportation Research Part A: General, vol. 25, no. 5, pp. 251-266, 1991.

[13] H. Yang, "Multiple equilibrium behaviors and advanced traveler information systems with endogenous market penetration," Transportation Research Part B: Methodological, vol. 32, no. 3, pp. 205-218, 1998.

[14] Y. Yin and H. Yang, "Simultaneous determination of the equilibrium market penetration and compliance rate of advanced traveler information systems," Transportation Research Part A: Policy and Practice, vol. 37, no. 2, pp. 165-181, 2003.

[15] M. Ben-Akiva and M. Bierlaire, "Discrete choice methods and their applications to short term travel decisions," in Handbook of Transportation Science, R. W. Hall, Ed., Springer, Berlin, Germany, 1999.

[16] E. Frejinger and M. Bierlaire, "Capturing correlation with subnetworks in route choice models," Transportation Research Part B: Methodological, vol. 41, no. 3, pp. 363-378, 2007.

[17] M. G. H. Bell and Y. Iida, Transportation Network Analysis, John Wiley \& Sons, Chichester, UK, 1997. 


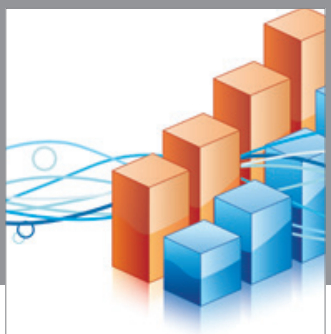

Advances in

Operations Research

vatem alat4

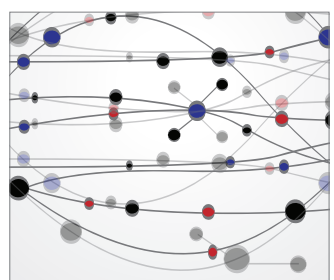

\section{The Scientific} World Journal
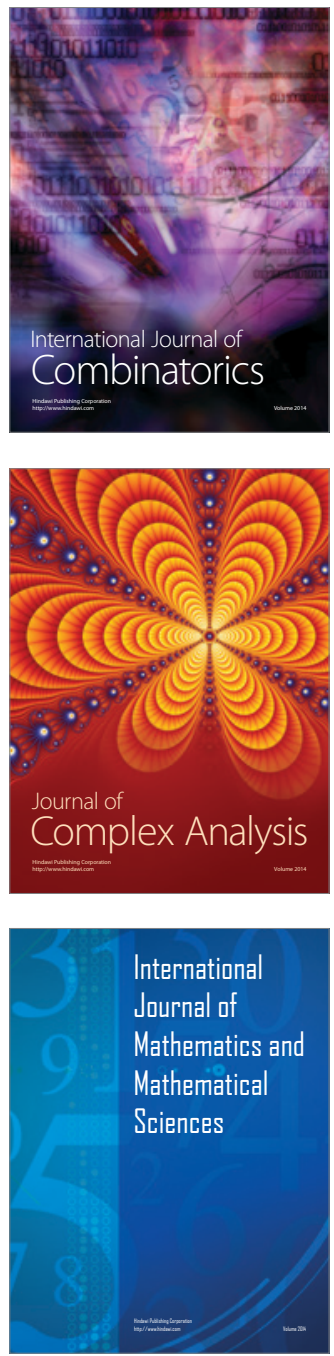
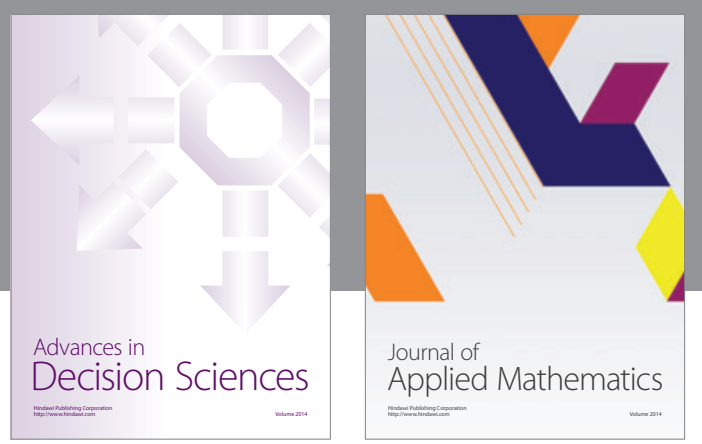

Algebra

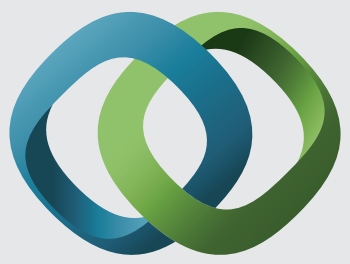

\section{Hindawi}

Submit your manuscripts at

http://www.hindawi.com
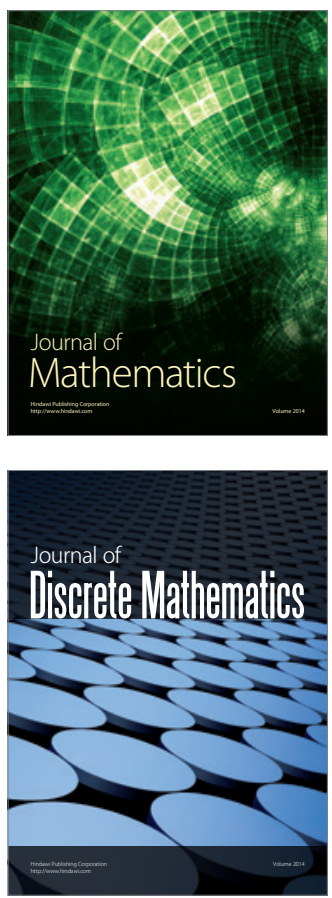

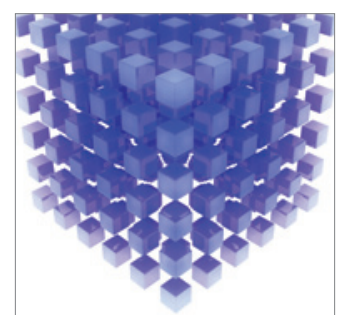

Mathematical Problems in Engineering
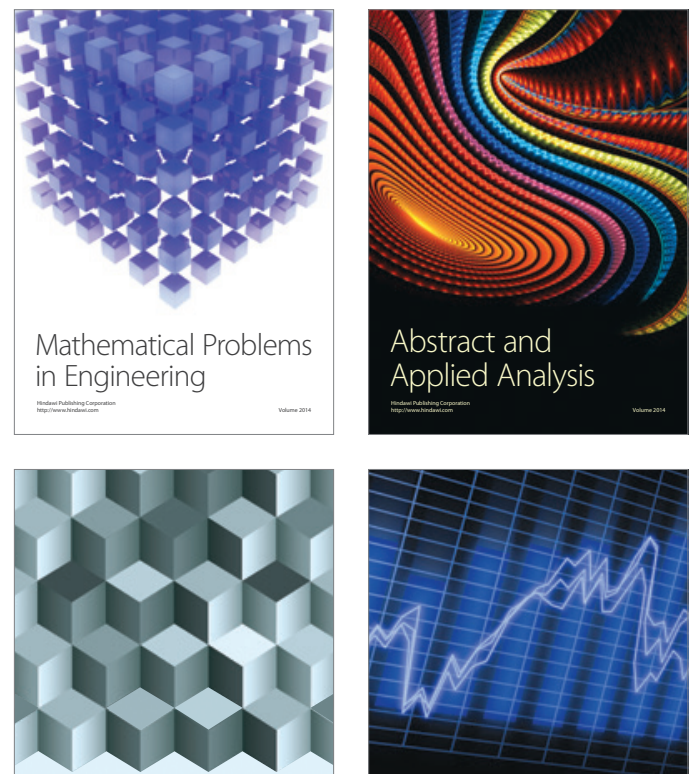

Journal of

Function Spaces

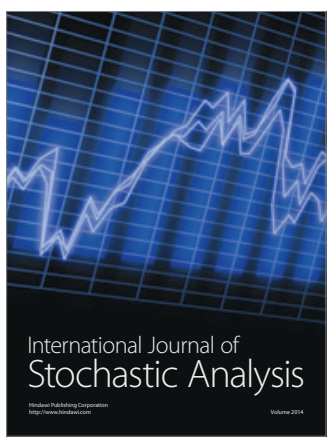

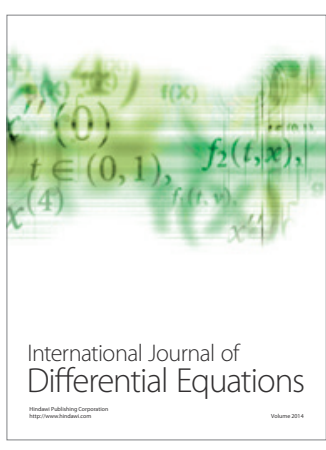
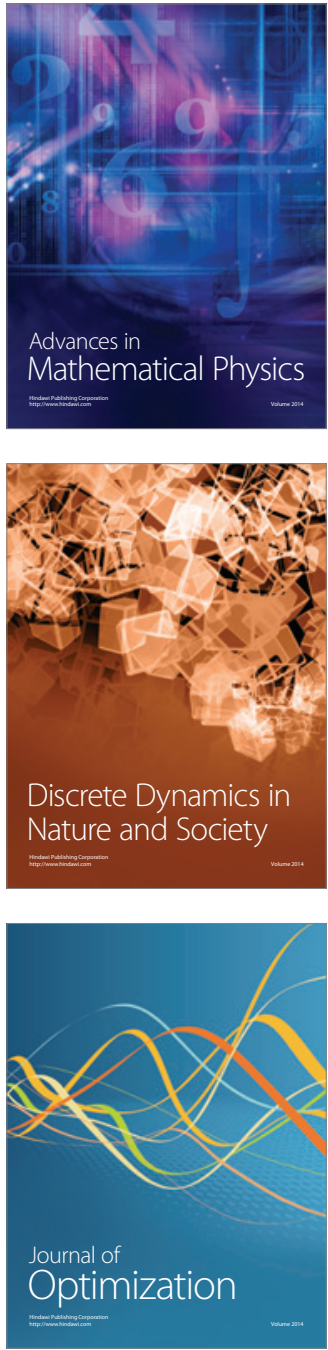